\title{
A COMPARATIVE STUDY BETWEEN EARLY VERSUS DEFERRED VITRECTOMY IN MANAGEMENT OF UNCOMPLICATED DIABETIC VITREOUS HAEMORRHAGE
}

\author{
Abd Ellateef, M. ${ }^{(*)}$, Abd El-Badie, M. \& Yossef, A. \\ Ophthalmology dept., Faculty of Medicine, Al-Azhar Univ., Assiut, Egypt \\ *E-mail: essam_ophth@yahoo.com
}

\begin{abstract}
Objective: To determine the visual outcome and frequency of complications after pars plana vitrectomy in cases of diabetic vitreous hemorrhage underwent early (within one month of onset) versus delayed vitrectomy (after one month of onset). Patients and Methods: Thirty eyes of 30 diabetic patients presented by uncomplicated diabetic vitreous haemorrhage divided into two groups; each group consists of fifteen eyes. Group Aincluded the patients presented by diabetic vitreous haemorrhage and undergone early vitrectomy within one month of onset. Group $B$ included the patients presented by diabetic vitreous haemorrhage after one month of onset and undergone delayed vitrectomy after one month of onset. Results: In group A, we found postoperative visual acuity ranges from $6 / 60$ to $6 / 12$ (1.00 to 0.30 Log MAR) with mean 0.58 ( Log $M A R)$. In group $B$ postoperative visual acuity ranges from $3 / 60$ to $6 / 18$ (1.30 to 0.48 Log MAR) with mean 0.75 ( Log MAR). Patients of type I DM underwent early vitrectomy have 0.62 (Log MAR) final mean BCVA, while those underwent delayed vitrectomy have 0.75 (Log MAR) final mean BCVA. P-value (0.04) shows statistical significance among both groups. Patients of type II DM underwent early vitrectomy have 0.56 (Log MAR) final mean BCVA, while those underwent delayed vitrectomy have 0.75 (Log MAR) final mean BCVA, P-value (0.42) shows statistical significance. The mean first day IOP was $19.13 \mathrm{mmHg}$ in group $A$ vs $17.8 \mathrm{mmHg}$ in group $B$. No patients in group $A$ had a first day $I O P$ recorded above $23 \mathrm{mmHg}$. In group $B$, however, two patients had a $1^{\text {st }}$ day IOP above $23 \mathrm{mmHg}$ and required a short course of oral acetazolamide and topical antiglaucoma medications. In contrast, three patients had postoperative hypotony $(I O P<10 \mathrm{mmHg})$ in group $A$ compared to one patient in group $B$. However, at the $1^{\text {st }}$ week follow-up appointment all IOPs had returned to within normal limits. P-value (0.364) shows no statistical significance among both groups Conclusion: Visual results after PPV in diabetic vitreous hemorrhage show that most of patients regain or retain useful vision. Early vitrectomy with endolaser retinal photocoagulation for diabetic vitreous haemorrhage (<30 days) decreases time spent with vision loss and the need for adjunctive PRP especially in patients of type I DM, recurrent vitreous haemorrhage and cases of premacular subhyaloid haem-orrhage. A few proportions of patients may develop late complications like recurrent vitreous hemorrhage and retinal detachment after successful PPV requiring secondary intervention.
\end{abstract}

Keywords: Diabetic retinopathy, Vitreous haemorrhage, Vitrectomy, Laser photocoagulation and anti-VEGF

\section{Introduction}

Diabetic retinopathy is a leading cause of blindness in the working popul- ation. With increasing duration and poor control of diabetes mellitus, microvascular 
complications can cause progressive retinal damage and lead to retinal ischemia, macular edema, retinal neovascularization, vitreous hemorrhage, traction retinal detachment, and neovascular glaucoma [1,2]. Primary management of diabetic retinopathy includes intensive glycemic control, as well as the regulation of systemic blood pressure and lipid levels. Improved blood glucose control can decrease the risk of the development and progression of diabetic retinopathy $[3,4]$. However, even with good medical management, $5 \%$ of patients will ultimately develop complications that require surgical intervention [5]. Refined technologies in vitrectomy surgery have allowed for improved instrumentation and surgical efficiency compared to prior 20-gauge PPV systems. The introduction of minimally invasive 23- and 25-gauge systems to the market, which is now considered as the standard in clinical practice, has permitted transconjunctival incisions and smaller sclerotomy size. Twenty-seven-gauge systems have also started to become available and may eventually become widely used in practice and earlier surgical intervention may be advisable [5]. Panretinal photocoagulation (PRP) is the first-line therapy for PDR. However, complications of PDR can arise before adequate PRP can be performed or even in the presence of seemingly adequate PRP. The goal of vitrectomy is to remove media opacity, relieve retinal traction (particularly macular traction), and perform PRP [6]. Additionally, removing the vitreous, and in particular the posterior hyaloid, also stabilizes the influence of PDR on the retina by removing the scaffold for fibrovascular proliferation. In theory, it may

\section{Patients and Methods}

This prospective comparative study was performed in Ophthalmology dept. of Al-Azhar university hospitals in the period from January 2016 to December 2018 and included 30 eyes of 30 diabetic patients presented by uncomplicated also improve blood vessel perfusion and oxygen supply to the inner retina $[7,8]$. The initial management of vitreous hemorrhage is typically to wait some time for spontaneous clearance, with application of adequate PRP to decrease VEGF loads and halt and/or regress fibrovascular proliferation. Vitrectomy with endolaser has usually been reserved for persistent and nonclearing vitreous hemorrhage lasting for more than three months or for recurrent vitreous hemorrhage despite prior PRP $[9,10]$. Longer observation periods may be permitted for patients with type 2 diabetes, due to increased rates of spontaneous resolution and slower progression of fibrovascular proliferation, especially in the presence of prior PRP or complete PVD. However, earlier surgical intervention may be warranted if there has been no previous PRP treatment performed or in the presence of extensive fibrovascular proliferation, rapid progression of retinopathy, anterior-segment neovascularization, or pre-existing visual loss in the fellow eye $[9,10]$. Additionally, the diabetic retinopathy vitrectomy study showed a benefit to early intervention in patients with type 1 diabetes, as these patients tend to have more quickly progressing retinopathy, and delay of adequate PRP may lead to the development of fibrovascular proliferation and increased risk of retinal detachment $[9,10]$. The aim of this work is to determine the visual outcome and frequency of complications after pars plana vitrectomy in diabetic vitreous hemorrhage cases underwent early versus delayed vitrectomy (after one month of onset).

diabetic vitreous haemorrhage. Approval of scientific committee of the Faculty of medicine, Al-Azhar university- Assiut, Egypt was obtained. A written informed consent was also obtained from each participant before the study. 


\subsection{Inclusion criteria}

Patients with diabetic retinopathy complicated by simple vitreous haemorrhage either presented recently (within

\subsection{Exclusion criteria}

Patients with other causes of vitreous haemorrhage e.g. traumatic, retinal breaks or retinal vein occlusion, patients with diabetic retinopathy complicated by vitreomacular traction, patients with rubeosis irides or neovascular glaucoma, previous

\subsection{Methods}

Institutional review board approval and complete informed consent was obtained for all patients. All patient underwent

\subsection{Preoperative data}

The collected preoperative data included: A) Patient age, gender, previous ocular surgery, previous argon laser retinal photocoagulation, preoperative intravitreal injections (including the type and time of injection), Hemoglobin A1c (HbA1c) concentration in the blood and coagulation profile to exclude other causes of haemorrhage and patient fitness for operation. B) Data recorded from the preoperative ophthalmic examination of the studied eye, which included: Best corrected visual acuity testing by Snellen acuity test, detailed Slit lamp (Topcon SL3F ${ }^{\mathrm{TM}}$, Japan) examination of anterior segment, iris and lens, followed by dilated posterior segment examination using double aspherical fundus lens +78D/ +90D (Volk ${ }^{\mathrm{TM}}$, USA), binocular indirect ophthalmoscope (Keeler ${ }^{\mathrm{TM}}$, Germany) with +20D (Volk, USA) lens, intraocular pressure (IOP) was measured by Goldmann applanation tonometer (Haag Streit ${ }^{\mathrm{TM}}$, Switzerland).

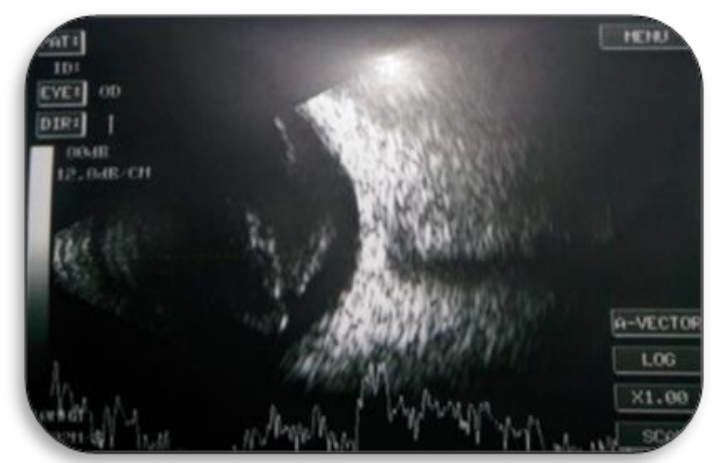

Intragel VH grade 2 one month of onset) or persistent nonclearing haemorrhage lasting more than one month.

vitrectomies (recurrent vitreous haemorhage after vitrectomy) and patients who had previous photocoagulation or intravitreal anti-VEGF injection within previous 3 months.

medical examination, and the collected data included preoperative data, surgical details and postoperative follow up data.

B-Scan (ocular ultrasonography) was done using (Sonomed ${ }^{\mathrm{TM}}$ ) B-Scan when fundus view was hazy, fig. (1). A scan U/S was done for all cases. Fundus fluorescein angiography and optical coherence tomography. Laboratory investigations like blood sugar level (fasting and random), glycosylated haemoglobin level (Hb A1C), virology screening (HBs Ag, Anti-HCV) were carried out. Radiological investigations like chest X-ray, electrocardiograpyh (ECG) and echocardiogram were done. The studied eyes were divided into 2 groups: the first group consists of 15 eyes of 15 diabetic patients, 9 males and 6 females, 9 type 2 $\mathrm{DM}$ and 6 type $1 \mathrm{DM}$, were underwent early vitrectomy within one month of onset. The second group consists of 15 eyes of 15 diabetic patients, 7 males and 8 females, 9 patients had type $2 \mathrm{DM}$ while 6 patients had type $1 \mathrm{DM}$.

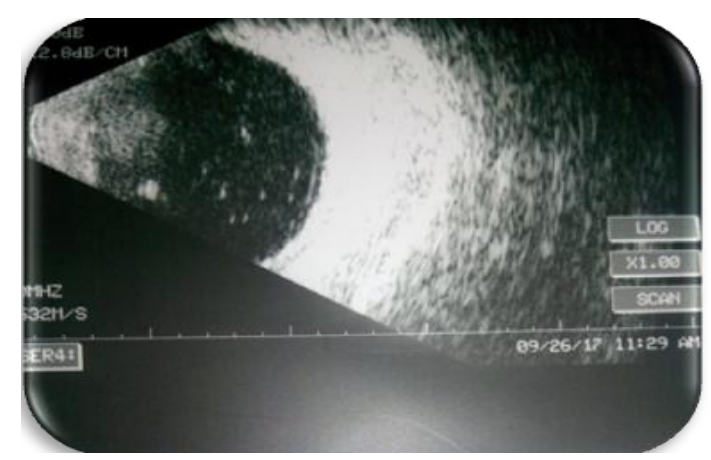

Intragel VH grade 3 


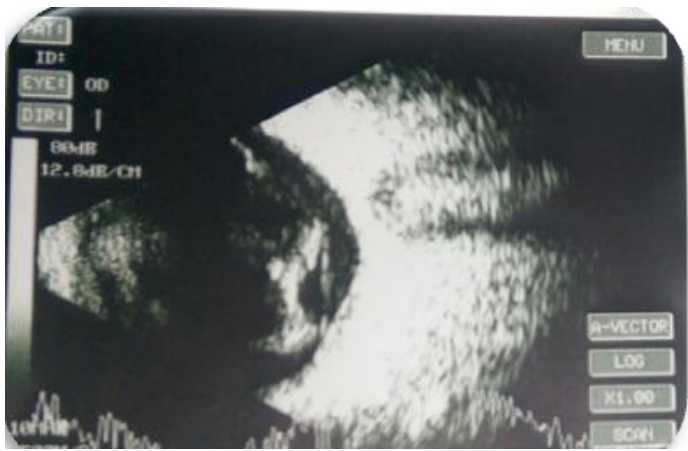

Intragel VH grade 2

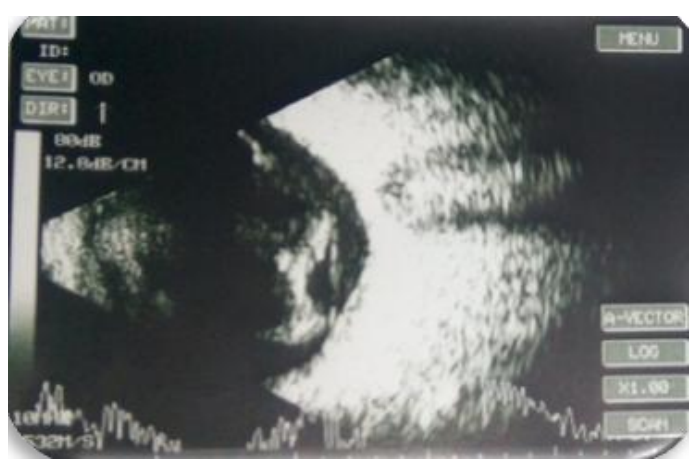

Intragel and subhyaloid $\mathrm{VH}$

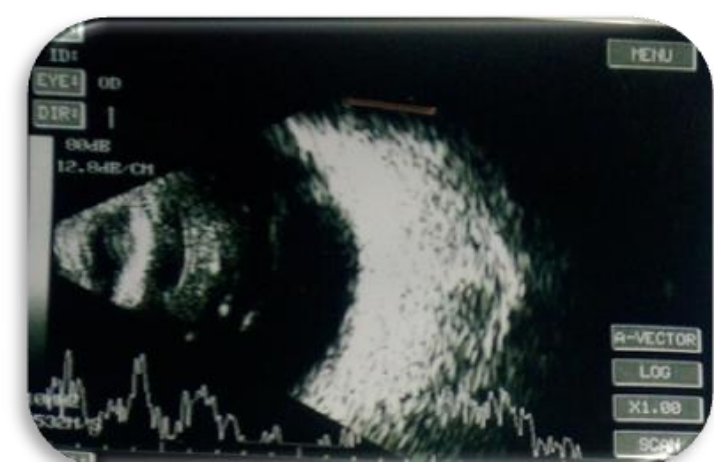

Persistant severe VH

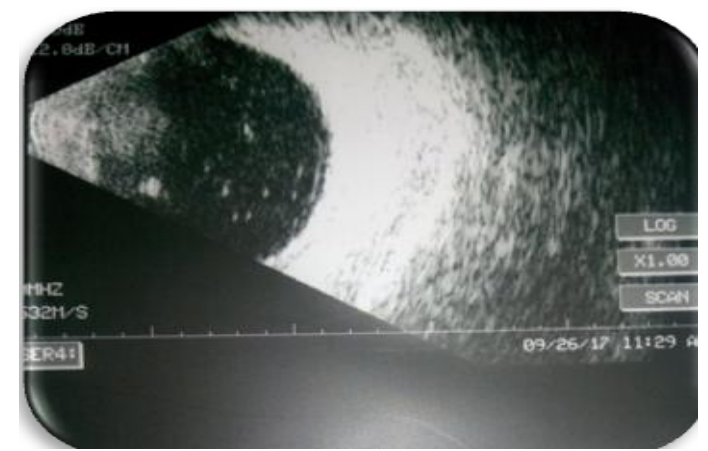

Intragel VH grade 3

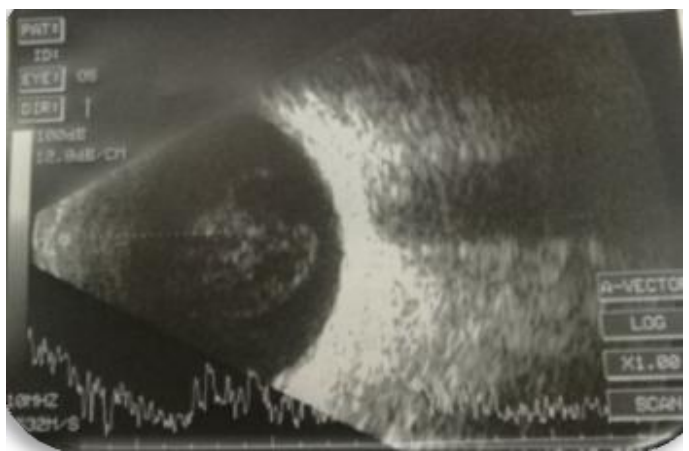

Persistant severe VH

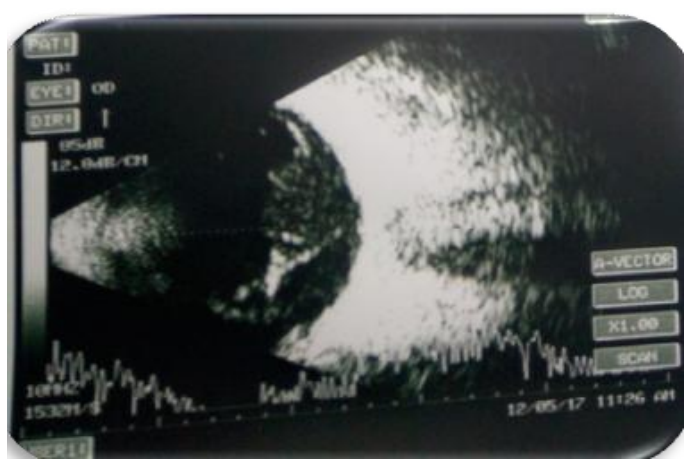

Severe subhyaloid VH

Figure (1) B-scan ultrasound for different degrees of vitreous haemorrhage.

\subsection{Instrumentation}

All eyes underwent standard 3-port pars plana vitrectomy using 23 -gauge $(23 \mathrm{G})$ instrumentation (Pharos ${ }^{\mathrm{TM}}$, Oertli, Swit-

\subsection{Surgical steps}

All patients underwent local monitored anaesthesia care. They underwent local monitored anaesthesia care and received peribulbaranaethesia. All eyes underwent standard 3-port PPV and dealing with retinal pathology according to the present situation using 23-gauge (23G) instrumentation (Pharos TM, Oertli, Switzerland) and using binocular indirect operating microscope system zerland) and using binocular indirect mphthalmo-microscopy system (BIOM).

(BIOM). Endolaserpanretinal photocoagulation (LIGHTMED TM diode endolaser, Switzerland) was applied to all eyes included in the study, fig. (2). In three patients when accidental lens touch occurred intraoperatively, phacoemulsification with implantation of foldable posterior chamber intraocular lens was done along with PPV. The vitreous cavity was filled with filtered air or saline 
in all patients except in two cases complicated by iatrogenic retinal holes or breaks, where silicon oil endotamponade was used. Patients were scheduled for follow up at
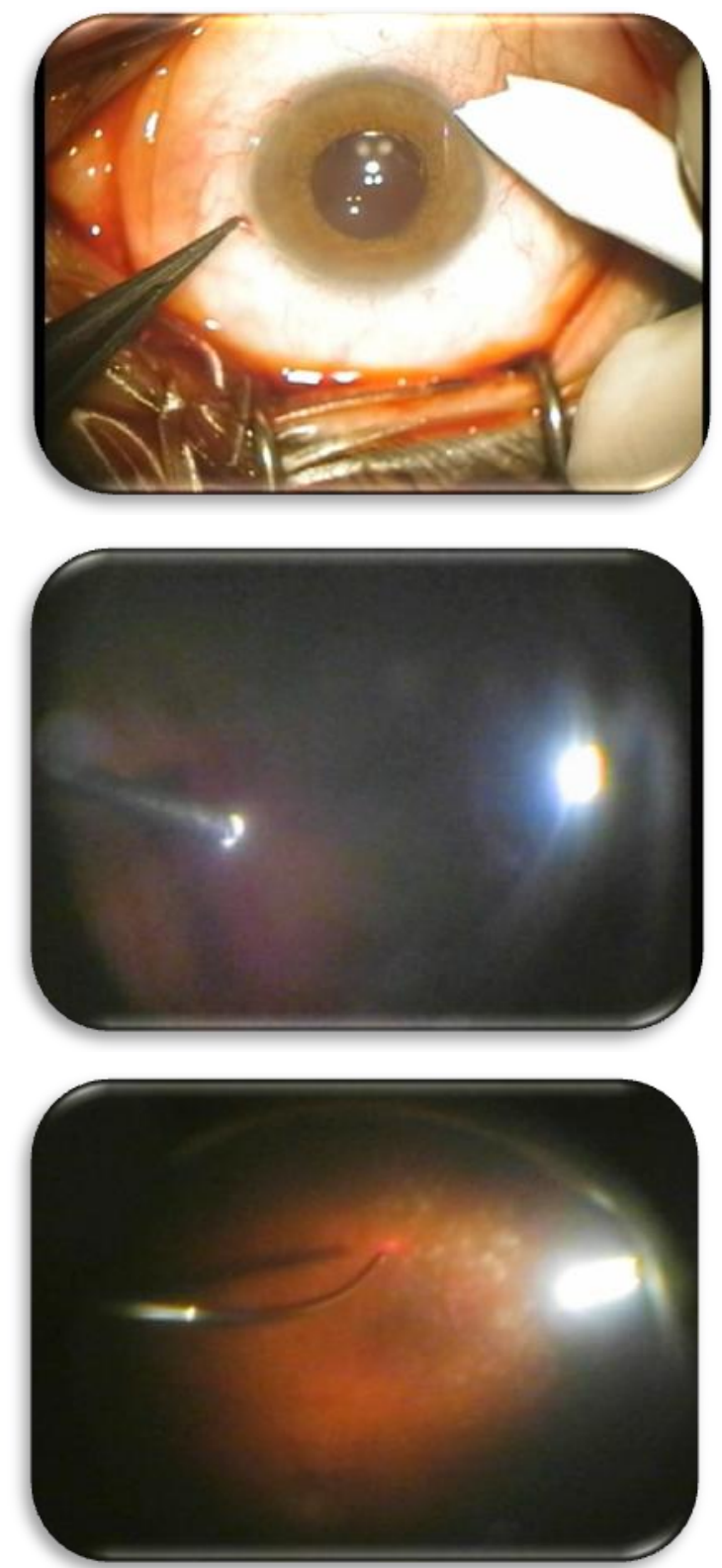

Figure (2) Operative procedures: Insertion of trocar cannulas, core vitrectomy, removal of vitreous base and endolaser photocoagulation.

\section{Statistical Analysis}

The collected data were revised, organized, tabulated and statistically analyzed using statistical package for social sciences (SPSS) version 20.0 for windows. Data are presented as the mean \pm standard deviation (SD), frequency, and percentage. Categorical variables were compared using the $1^{\text {st }}$ day to evaluate serious adverse effect like endophthalmitis and IOP elevation. Then at $1^{\text {st }}$ week, $3^{\text {rd }}$ week, 1 month and three months postoperatively.
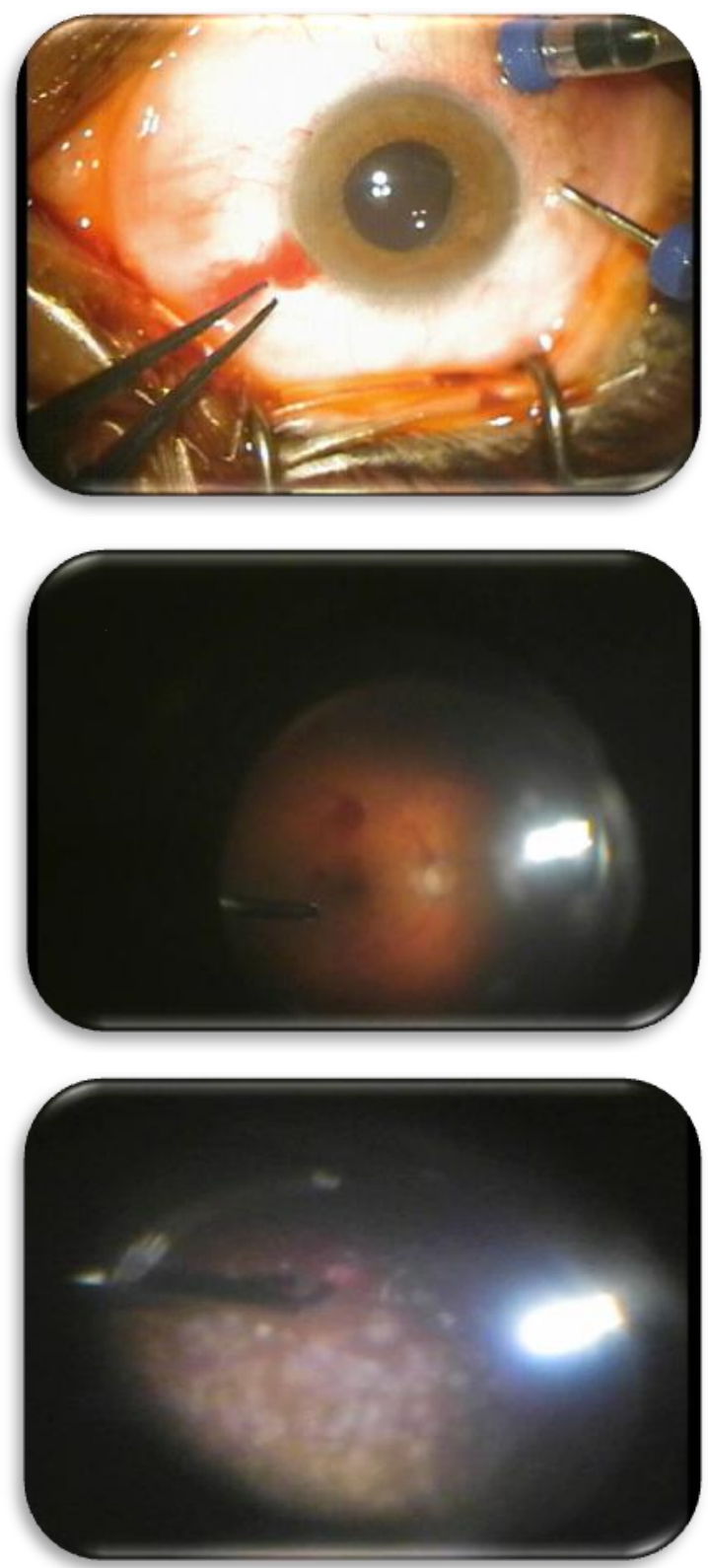

-square $\left(\chi^{2}\right)$ test and Fisher's exact test (if required). Continuous normally distributed data were compared by the independent sample $t$ test (two-tailed) and paired sample $t$ test to detect the differences between the studied groups. The level of signifycance was accepted if the $\mathrm{P}$ value $<0.05$. 


\section{Results}

\subsection{Patient demographics}

The following tables show demographic distribution of age and sex in both groups. The mean age of group A was $40.6 \pm$ 8.17 years, while for group B was 52.6 \pm 7.58 years. Group A included 9 males

and 6 females, while group B included 7 males and 8 females. P-value (0.335) shows no statistical significance, $\operatorname{tab}_{\mathrm{s}}$. (1 \& 2).

Table (1) Age distribution among both groups.

\begin{tabular}{|l|c|c|}
\cline { 2 - 3 } \multicolumn{2}{c|}{} & \multicolumn{2}{c|}{ Group A } & Group $B$ \\
\hline Range & $32-48$ years & $45-59$ years \\
\hline Mean & 40.6 & 52.6 \\
\hline +SD & \pm 8.17 & \pm 7.58 \\
\hline t. test & \multicolumn{2}{|c|}{-0.980} \\
\hline p. value & \multicolumn{2}{|c|}{0.335} \\
\hline
\end{tabular}

Table (2) Gender distribution among both groups.

\begin{tabular}{|l|c|c|}
\cline { 2 - 3 } \multicolumn{2}{c|}{ Male } & Female \\
\hline Group A & $9(60 \%)$ & $6(40 \%)$ \\
\hline Group B & $8(53.3)$ & $7(46.7 \%)$ \\
\hline Total & $17(56.7 \%)$ & $13(43.3 \%)$ \\
\hline p. value & \multicolumn{2}{|c|}{0.500} \\
\hline
\end{tabular}

\subsection{Preoperative visual acuity in both groups}

Visual acuity ranges from $\mathrm{HM}$ to $2 / 60$

(2.3 to 1.48 LogMAR) with mean preoperative visual acuity 2.00 (LogMAR) in group A. While visual acuity ranges from

HM to $3 / 60$ (2.3 to $1.30 \operatorname{LogMAR}$ ) with mean preoperative visual acuity 1.88 (Log MAR) in group B.P-value (0.500) shows no statistical significance, tab. (3).

Table (3) Preoperative visual acuity in both groups.

\begin{tabular}{|c|c|c|}
\hline Preoperative BCVA & Group A & Group B \\
\hline Range & $\mathrm{HM}$ to $2 / 60$ & HM to $3 / 60$ \\
\hline Mean LogMAR & 2.00 & 1.88 \\
\hline \pm SD & \pm 0.31243 & \pm 0.3499 \\
\hline Independent $\mathrm{t}$ test & \multicolumn{2}{|c|}{0.958} \\
\hline p. value & \multicolumn{2}{|c|}{0.346} \\
\hline
\end{tabular}

\subsection{Duration of diabetes}

Duration of diabetes ranges from 8

9 to 18 with mean 12.86 years in group to 22 years with mean 11.46 years in group A. While duration of diabetes ranges from B.P-value (0.222) shows no statistical significance, tab. (4).

Table (4) Duration of diabetes.

\begin{tabular}{|r|c|c|}
\hline \multirow{2}{*}{ Range } & Group A & Group B \\
\hline Mean & $8-22$ years & $9-18$ years \\
\hline +SD & 11.46 & 12.86 \\
\hline t. test & \pm 3.48193 & \pm 2.58752 \\
\hline p. value & \multicolumn{2}{|c|}{-1.250} \\
\hline \multicolumn{3}{|c|}{0.222} \\
\hline
\end{tabular}




\subsection{Type of diabetes}

Twelve cases included in our study were type I insulin dependent DM (40\%), while 16 patients were type II non-insulin dependent DM (60\%). Six cases of type I DM patients undergone early vitrectomy (group A) and the others undergone delayed vitrectomy. Nine cases of type II DM patients undergone early vitrectomy (group A) and the others undergone delayed vitrectomy, tab. (5).

Table (5) Type of diabetes

\begin{tabular}{|c|c|c|c|}
\hline Type of DM & Number & Group A & Group B \\
\hline Type I & $12(40 \%)$ & $6(20 \%)$ & $6(20 \%)$ \\
\hline Type II & $18(60 \%)$ & $9(30 \%)$ & $9(30 \%)$ \\
\hline
\end{tabular}

\subsection{Medical and ophthalmic history}

Medical history of patients included in the study reveals that 2 patients treated medically by only oral hypoglycaemic drugs (6.6\%), five patients treated by oral hypoglycaemic drugs and diet regulation (16.6\%), six patients treated by oral hypoglycaemic drugs and insulin (20\%), while 17 patients were treated by insulin only $(56.6 \%)$. All cases received argon laser photocoagulation

in the previous three months of presentation were excluded from the study, beyond this period were 8 patients included (26.6\%). Thirteen patients were treated by intravitreal injection of anti-VEGF (43.3\%). Seven patients received only single injection (23.3\%), four patients received two injections (13.3\%), while the other two patient received more than 2 injections (6.6\%), tab. (6).

Table (6) Medical and ophthalmic history

\begin{tabular}{|c|c|c|c|}
\hline Treatment & \multicolumn{2}{|l|}{ History } & Total \\
\hline \multirow{4}{*}{ Medical Treatment } & \multicolumn{2}{|l|}{ Diet + Oral } & $5(16.6 \%)$ \\
\hline & \multicolumn{2}{|l|}{ Oral } & $2(6.6 \%)$ \\
\hline & \multicolumn{2}{|l|}{ Oral + Insulin } & $6(20 \%)$ \\
\hline & \multicolumn{2}{|l|}{ Insulin } & $17(56.6 \%)$ \\
\hline \multirow{4}{*}{$\begin{array}{l}\text { Argon Laser (All cases had laser } \\
\text { sessions of durations more than three } \\
\text { months before haemorrhage occurred) }\end{array}$} & \multirow{2}{*}{ Previous Laser } & Yes & $8(26.6 \%)$ \\
\hline & & No & $22(73.3 \%)$ \\
\hline & \multirow{2}{*}{$\operatorname{Sessios}(\mathrm{n}=8)$} & Single & $7(23.3 \%)$ \\
\hline & & Multiple & $1(3.3 \%)$ \\
\hline \multirow{5}{*}{ Intravitreal Injection } & \multirow{2}{*}{ Previous Injection } & Yes & $13(43.3 \%)$ \\
\hline & & No & $17(56.6 \%)$ \\
\hline & \multirow{3}{*}{ Number of Injections $(n=43)$} & One & $7(23.3 \%)$ \\
\hline & & Two & $4(13.3 \%)$ \\
\hline & & $>2$ & $2(6.6 \%)$ \\
\hline
\end{tabular}

\subsection{Postoperative visual acuity}

Twenty three cases have regained BCVA >1.00 Log MAR (76.6\%), 13 cases in group A (43.3\%) and 12 cases in group $\mathrm{B}(33.3 \%)$. Three cases have $\mathrm{BCVA}=1.00$ Log MAR (10\%), one in group A $(3.3 \%)$ and two in group B $(6.6 \%)$. While four cases have BCVA<1.00 Log MAR (13.3\%), one in group A $(3.3 \%)$ and three $(10.0 \%)$ in group B. In group A, postoperative visual acuity ranges from $6 / 60$ to $6 / 12$ (1.00 to 0.30 Log MAR) with mean 0.57 (Log MAR). In group $B$ postoperative visual acuity ranges from $3 / 60$ to $6 / 18$ (1.30 to 0.48
Log MAR) with mean 0.76 (Log MAR). Patients of type I DM who underwent early vitrectomy achieved a mean BCVA 0.58 (Log MAR) at the end of follow-up period in comparison of a mean $0.76(\mathrm{Log}$ MAR) for patients who underwent delayed vitrectomy .Patients of type II DM who underwent early vitrectomy achieved a mean BCVA 0.57 (Log MAR) at the end of follow-up period in comparison of a mean 0.77 (Log MAR) who underwent delayed vitrectomy, tabs. (7-11). 
Table (7) Postoperative visual acuity among both groups.

\begin{tabular}{|l|c|c|}
\multicolumn{1}{|c|}{ Postoperative BCVA } & Group A & Group B \\
\hline Range & $6 / 60$ to 6/12 & $3 / 60$ to 6/18 \\
\hline Mean LogMar & 0.583 & 0.751 \\
\hline + SD & \pm 0.2661 & \pm 0.2597 \\
\hline Indep. t test & \multicolumn{2}{|c|}{-0.750} \\
\hline p. value & \multicolumn{2}{|c|}{0.04} \\
\hline
\end{tabular}

Table (8) Pre - and postoperative BCVA among both groups.

\begin{tabular}{|lc|c|c|c|} 
& \multicolumn{2}{c}{ Early vitrectomy, Group A } & \multicolumn{2}{c|}{ Delayed vitrectomy, Group B } \\
\cline { 2 - 5 } & Preop. $\boldsymbol{V A}$ & Postop. $\boldsymbol{B C} \boldsymbol{C} \boldsymbol{A}$ & Preop. $\boldsymbol{V A}$ & Postop. BCVA \\
\hline \multirow{3}{*}{ Range } & HM to $2 / 60$ & $4 / 60$ to $6 / 12$ & HM to $3 / 60$ & $6 / 60$ to $6 / 24$ \\
& 2.30 to $1.48 \mathrm{Log}$ & 1.18 to $0.3 \mathrm{Log}$ & 2.30 to $1.30 \mathrm{Log}$ & 1.0 to $0.60 \mathrm{Log}$ \\
& $\mathrm{MAR}$ & MAR & MAR & MAR \\
\hline Mean $\mathbf{\text { SD }}$ & $2 \pm 0.31$ & $0.57 \pm 0.26$ & $1.88 \pm 0.35$ & $0.76 \pm 0.25$ \\
\hline Indep. t test & 0.958 & -0.760 & 0.958 & -0.760 \\
\hline p. value & 0.346 & 0.043 & 0.346 & 0.049 \\
\hline
\end{tabular}

Table (9) Detailed pre- and postoperative Log MAR BCVA

\begin{tabular}{|l|c|c|c|c|}
\multicolumn{1}{|c}{ BCVA Log MAR (Snellen) } & Preoperative n (\%) & Group A & Group B & Total \\
\hline $\mathbf{0 . 3 0}(\mathbf{6} / \mathbf{1 2})$ & --- & $3(10 \%)$ & --- & $3(10 \%)$ \\
\hline $\mathbf{0 . 4 8}(\mathbf{6} / \mathbf{1 8})$ & --- & $5(16.6 \%)$ & $3(10 \%)$ & $8(26.6 \%)$ \\
\hline $\mathbf{0 . 6 0}(\mathbf{6} / \mathbf{2 4})$ & --- & $4(13.3 \%)$ & $4(13.3 \%)$ & $8(26.6 \%)$ \\
\hline $\mathbf{0 . 7 8}(\mathbf{6 / 3 6})$ & --- & $1(3.3 \%)$ & $3(10 \%)$ & $4(13.3 \%)$ \\
\hline $\mathbf{1 . 0 0}(\mathbf{6 / 6 0})$ & --- & $1(3.3 \%)$ & $2(6.6 \%)$ & $3(10 \%)$ \\
\hline $\mathbf{1 . 0 8}(\mathbf{5 / 6 0})$ & --- & --- & $2(6.6 \%)$ & $2(6.6 \%)$ \\
\hline $\mathbf{1 . 1 8}(\mathbf{4 / 6 0})$ & --- & $1(3.3 \%)$ & $1(3.3 \%)$ & $2(6.6 \%)$ \\
\hline $\mathbf{1 . 3 0}(\mathbf{3 / 6 0})$ & $1(3.3 \%)$ & --- & --- & --- \\
\hline $\mathbf{1 . 4 8}(\mathbf{2 / 6 0})$ & $5(16.6 \%)$ & --- & --- & --- \\
\hline $\mathbf{1 . 7 7}(\mathbf{1 / 6 0})$ & $3(10 \%)$ & --- & --- & -- \\
\hline $\mathbf{1 . 8 5}(\mathbf{C F})$ & $9(30 \%)$ & --- & --- & --- \\
\hline $\mathbf{2 . 3 0}(\mathbf{H M})$ & $12(40 \%)$ & --- & --- & --- \\
\hline Mean BCVA & & 0.57 & 0.76 & \\
\hline Total & $30(100 \%)$ & & $30(100 \%)$ & \\
\hline
\end{tabular}

Table (10) Postoperative visual acuity among both groups and types of diabetes.

\begin{tabular}{|c|c|c|c|c|}
\hline & \multicolumn{2}{|c|}{ Early vitrectomy, Group A } & \multicolumn{2}{|c|}{ Delayed vitrectomy, Group B } \\
\hline & Range & $M e a n+S D$ & Range & $M e a n+S D$ \\
\hline Type I DM & $6 / 60$ to $6 / 24$ & $0.62 \pm 0.241$ & $4 / 60$ to $6 / 24$ & $0.75 \pm 0.196$ \\
\hline Type II DM & $4 / 60$ to $6 / 12$ & $0.56 \pm 0.274$ & $5 / 60$ to $6 / 24$ & $0.75 \pm 0.205$ \\
\hline
\end{tabular}

Table (11) BCVA according to type of DM

\begin{tabular}{|c|c|c|c|c|}
\hline \multirow{2}{*}{$\begin{array}{l}\text { Log MAR } \\
\text { BCVA }\end{array}$} & \multicolumn{2}{|c|}{$\begin{array}{c}\text { Type I (14 cases) } \\
\text { Mean (BCVA } 0.67)\end{array}$} & \multicolumn{2}{|c|}{$\begin{array}{c}\text { Type II (16 cases) } \\
\text { Mean (BCVA } 0.67)\end{array}$} \\
\hline & Group A (7 cases) & Group B (7 cases $)$ & Group A (8 cases) & Group B (8 cases) \\
\hline 0.30 & 1 & --- & 2 & --- \\
\hline 0.48 & 2 & 1 & 3 & 2 \\
\hline 0.60 & 3 & 2 & 1 & 2 \\
\hline 0.78 & --- & 2 & 1 & 1 \\
\hline 1.00 & 1 & 1 & --- & 1 \\
\hline 1.08 & --- & 1 & $\begin{array}{ll}-- \\
\end{array}$ & 1 \\
\hline 1.18 & --- & --- & 1 & 1 \\
\hline Mean & 0.58 & 0.76 & 0.57 & 0.77 \\
\hline P-value & \multicolumn{2}{|c|}{0.042} & \multicolumn{2}{|c|}{0.048} \\
\hline
\end{tabular}




\subsection{Pre-and postoperative IOP measurements}

The mean preoperative IOP was $16.6 \pm 0.31 \mathrm{mmHg}$ in group A vs. $17.88 \pm$ $0.35 \mathrm{mmHg}$ in group $\mathrm{B}$. The mean first day postoperative IOP was $19.13+4.01 \mathrm{mmHg}$ in group A vs. $17.8+2.69 \mathrm{mmHg}$ in group B. One patient in group A had a first day IOP recorded above $23 \mathrm{mmHg}$. In group $\mathrm{B}$, however, two patients had a $1^{\text {st }}$ day
IOP above $23 \mathrm{mmHg}$ and required a short course of oral acetazolamide and topical antiglaucoma medications. In contrast, three patients had postoperative hypotony (IOP $<9 \mathrm{mmHg}$ ) in group A compared to one patient in group B. However, all the patients had an IOP within normal range at the end of the $1^{\text {st }}$ week post-operatively, tab. (12).

Table (12) Statistical analysis of the results of IOP measurements.

\begin{tabular}{|lc|c|c|c|}
\multicolumn{1}{c}{} & \multicolumn{2}{c}{ Early vitrectomy - Group A } & \multicolumn{2}{c|}{ Delayed vitrectomy - Group B } \\
\cline { 2 - 5 } & Preop. IOP & Postop. IOP & Preop.IOP & Postop. IOP \\
\hline Range & 16 to $25 \mathrm{mmHg}$ & 15 to $23 \mathrm{mmHg}$ & 15 to 27 & 15 to $28 \mathrm{mmHg}$ \\
\hline Indep. t test & $16.6 \pm 0.31$ & $19.13 \pm 4.01$ & $17.88 \pm 0.35$ & $17.86 \pm 2.69$ \\
\hline p. value & 0.958 & 1.014 & 0.958 & 1.012 \\
\hline
\end{tabular}

\subsection{Postoperative complications}

Lens touch was occurred in only one case in group A (3.3\%), while occurred in two cases in group B $(6.6 \%)$. Iatrogenic retinal break was occurred in two cases $(6.6 \%)$, one in each group (3.3\%). All are treated intraoperatively by laser photocoagulation and SO endotamponade. Intraoperative corneal oedema was occurred in three cases in group A (10\%), while occurred in two cases in group B (6.6\%). All cases were treated by removal of epithelium to enhance the visualization. Postoperative hypotony was occurred in only one case in group B (3.3\%), while occurred in three cases in group A $(10 \%)$. It could be due to wound leak from suturelesssclerotomy sites. Postoperative elevated IOP was occurred in two cases in group B $(6.6 \%)$, while occurred in only one case in group A $(3.3 \%)$. All cases were treated by short course of antiglaucoma drugs including systemic and topical acetazolamide. Recurrent vitreous haemorrhage was occurred in two cases $(6.6 \%)$, one in each group (3.3\%). It could be due to insufficient photocoagulation, retinal break or bleeding from sclerotomy ports. One case needed secondry intervention while the other was minimal bleeding which resolved spontaneously after two weeks. Postvitrectomy retinal detachment was occurred in two cases $(6.6 \%)$, one in each group $(3.3 \%)$. It can be caused by iatrogenic retinal tear, atrophic retinal holes, late contractions of vitreous incarceration and peripheral traction due to anterior hyaloid proliferation. Both cases undergone secondary intervention and treated by laser photocoagulation and silicon oil endotamponade. Phacoemulsification and silicon oil removal was done after 3 months and the retina still attached after silicon oil removal. Cataract formation was occurred in two cases in group A (6.6\%), while occurred in three cases in group B (10\%).Two cases were complicated to silicon oil. All cases are treated by phacoemulsification. Neovascular glaucoma was occurred in one case in group A (3.3\%), while occurred in two cases in group B (6.6\%). One case of them are one of those undergone combined phacoemulsification due to accidental lens touch, tab. (13). 
Table (13) Postoperative complications among both groups.

\begin{tabular}{|c|c|c|}
\hline Complications & $(\operatorname{Group} A)$ & (Group B ) \\
\hline Lens touch & 1 case $(3.3 \%)$ & 2 cases $(6.6 \%)$ \\
\hline Iatrogenic retinal break & 1 case $(3.3 \%)$ & 1 case $(3.3 \%)$ \\
\hline Intra operative corneal oedema & 3 cases $(10 \%)$ & 2 cases $(6.6 \%)$ \\
\hline Postoperative hypotony & 3 case $(10 \%)$ & 1 cases $(3.3 \%)$ \\
\hline Postoperative elevated IOP & 1 case $(3.3 \%)$ & 2 cases $(6.6 \%)$ \\
\hline Recurrent vitreous haemorrhage & 1 case $(3.3 \%)$ & 1 case $(3.3 \%)$ \\
\hline Postvitrectomy retinal detachment & 1 case $(3.3 \%)$ & 1 case $(3.3 \%)$ \\
\hline Cataract formation & 2 cases $(6.6 \%)$ & 3 cases $(10 \%)$ \\
\hline Neovascular glaucoma & 1 case $(3.3 \%)$ & 2 cases $(6.6 \%)$ \\
\hline
\end{tabular}

\section{Discussion}

Visual outcome after successful PPV in diabetic vitreous hemorrhage depends on the macular function. Recent evidence suggest better outcome in VA when compared with the earlier studies, which be due to improved diabetes control and its systemic co-morbidities, better surgical techniques, early diagnosis and management and surgeons experience and confidence [11]. In type $1 \mathrm{DM}$, the frequency of PDR increases up to $41 \%$ when the duration of diabetes exceeds 15 years. In type 2 $\mathrm{DM}$, on the other hand, the rate of PDR is $3 \%$ when the duration of the disease exceeds 10 years. In our study $40 \%$ of patients are type $1 \mathrm{DM}$ while $60 \%$ are type $2 \mathrm{DM}$ and $53 \%$ of our patients have 11-15 years duration of diabetes mellitus. A study by Guzey et al reported type $1 \mathrm{DM}$ in $17.3 \%$, type $2 \mathrm{DM}$ in $82.6 \%$ and mean duration of diabetes was $19.4 \pm 4.9$ and $15.3 \pm 7.4$ years respectively [12]. Qamar et al reported type $1 \mathrm{DM}$ in $14.6 \%$, type $2 \mathrm{DM}$ in $85.3 \%$ and mean duration of diabetes was 14 years and 11 years respectively [13]. Although panretinal photocoagulation is considered the first line treatment for PDR, anti-proliferative drugs (anti-VEGF) are slowly finding their place in the management [14]. The objective of preoperative laser photocoagulation and anti- VEGF agents alone or in combination is to facilitate the control of the vascular proliferative process [11]. In our study, $26.6 \%$ patients have laser photocoagulation, $23.3 \%$ have single session PRP and $3 \%$ have multiple PRP sessions. Pre-operative PRP was rep- orted in $60 \%$ by Mason et al [15], 72\% by Ünver et al [16] and $47.6 \%$ by Qamar et al. [13]. Anti-VEGF agents reduce the time required for vitreous clear-up and decrease the need for vitrectomy [17]. In our study $43 \%$ of (13) patients have previous anti-VEGF injection, seven patients received only single injection (23.3\%), four patients received two injections $(13.3 \%)$, while the other two patient received more than 2 injections $(6.6 \%)$. The outcome of PPV in eyes where prior intensive laser photocoagulation and Anti- VEGF agents have been applied are more satisfactory [18]. Despite the intensive photocoagulation applied preoperatively, the proliferations are observed to continue in $73 \%$ of cases of the study. At this stage, PPV and additional intraoperative endolaser photocoagulation are necessary. In June 2015 Janelle et al conducted a retrospective study to compare early ( $<30$ days) versus delayed (>30 days) vitrectomy for the management of vitreous hemorrhage due to PDR and suggested that early vitrectomy for $\mathrm{VH}$ due to PDR significantly decreases time spent with vision loss, and decreases the need for further PRP sessions [19]. The rationale of this study was the concept that pars plana vitrectomy improves visual acuity in patients with diabetic vitreous hemorrhage. Therefore we conducted this study to determine visual outcome after pars plana vitrectomy in diabetic vitreous hemorrhage and to determine the frequency of complications after pars plana vitrectomy in diabetic vitreous haemorrhage. Surgery for vitreous hemorrhage without macular 
detachment produces, in most of cases, good visual acuity. In our study $83.3 \%$ of cases have final Log MAR BCVA > 1.00. Twenty three cases have regained BCVA > 1.00 Log MAR (76.6\%), 13 cases in Group A (43.3\%) and 10 cases in group B $(33.3 \%)$. Three cases have BCVA= 1.00 Log MAR (10\%), one in group A and 2 two in group B. While four cases have BCVA<1.00 Log MAR (13.3\%), three of them were in the delayed vitrectomy group, group B and only one case in group A. The mean final postoperative BCVA was $0.58 \pm 0.26$ in group $\mathrm{A}$, while was $0.75 \pm$ 0.25 in group B with significant statistical difference between the two groups ( $p$. value 0.04 ). The mean visual outcome was better in the early vitrectomy group. Also, patients of type I DM undergone early vitrectomy have 0.62 (Log MAR) final mean BCVA, while those undergone delayed vitrectomy have 0.75 (Log MAR) final mean BCVA with significant statistical difference between the two groups. Patients of type II DM undergone early vitrectomy have 0.56 (Log MAR) final mean BCVA, while those undergone delayed vitrectomy have 0.75 (Log MAR) final mean BCVA. The results shows that patients of type I DM regain a better vision when undergo early vitrectomy than those undergone late vitrectomy. Early vitrectomy helps in improving and regains the macular function, decreasing retinal ischaemia and further neovascular proliferation and decreasing the incidence of photoreceptor damage, haemosiderosis bulbi and haemolytic glaucoma. On the other hand permanent impairment of visual acuity was more in the delayed vitrectomy group .Four cases have BCVA $<1.00$ Log MAR $(13.3 \%)$, three of them were in the delayed vitrectomy group, group B and only one case in group A. Two of them were premacular subhyaloid haemorrhage, one case was that complicated by $\mathrm{RD}$ and the fourth case was with ischaemic maculopathy. Further investigations performed (FFA \& OCT) to determine

\subsection{Postoperative complications after PPV for diabetic vitreous haemorrhage}

Postoperative complications after

PPV for diabetic vitreous haemorrhage includes post vitrectomy recurrent vitreous hemorrhage, cataract formation, postvi- the causes of visual impairment after vitrectomy which were macular ischemia, macular edema, RD and neovascular glaucoma. Cases of premacular subhyaloid haemorrhage should undergo early vitrectomy to avoid photoreceptor damage and macular ischaemia caused by perisistant vitreous haemorrhage. On the other hand three cases of premacular subhyaloid haemorrhage included in early vitrectomy group have regained BCVA Log MAR 0.30 (6/12), Log MAR 0.48 (6/18) and Log MAR 0.78 (6/36). Gupta et al [20] reported final visual acuity of Log MAR $\geq 0.3$ in $65 \%$ cases. Another study by of $\log$ MAR $\leq 0.3$ in $50.9 \%$ cases. In our study mean pre \& post-operative Log MAR BCVA is 1.016 and 0.74 respectively. Gupta et al reported mean pre \& postoperative Log MAR BCVA as 1.56 and 0.66 respectively [20]. This difference is due to small sample size and short follow up period in our study. Overall, these results are an improvement from diabetic retinopathy vitrectomy study (DRVS) where $33.3 \%$ achieved final $\mathrm{BCVA} \leq 0.48 \mathrm{Log}$ MAR (6/18) after PPV for vitreous hemorrhage. Due to early diagnosis and earlier intervention pre-operative visual acuity improved with time and this correlates with improved post-operative visual results. Patients with poor pre-operative visual acuity although achieve greater improvement in log MAR but still has worse final post-operative visual acuity. The Early Treatment Diabetic Retinopathy Study (ETDRS) suggested that the results of surgery were better with earlier vitrectomy. As with time the surgical methods has changed significantly since the ETDRS and there can be a justification for repeating that study with modern methods. Due to improvement in vitreoretinal surgery, the outcome after diabetic vitrectomy continues to improve [21].

trectomy retinal detachment, neovascular glaucoma, postoperative hypotony, postoperative elevated IOP and postoperative endophthalmitis. Gupta et al [14] reported final visual acuity 


\subsection{Post vitrectomy recurrent vitreous hemorrhage}

Vitreous cavity hemorrhage following PPV for vitreous hemorrhage has been reported in $7 \%$ to $63 \%$ of patients [22-30]. Vitreous cavity hemorrhage following PPV can be present from the first postoperative day (persistent-20\% to $63 \%$ of patients), or can occur within the first 4 to 6 weeks (early-5\%), or thereafter (delayed-8\%) [22-29]. Persistent and early vitreous cavity hemorrhage are mainly secondary to incomplete intraoperative hemostasis, bleeding from dissected fibrovascular tissue and release of erythrocytes from residual peripheral vitreous gel and iatrogenic injury to the retina or retinal vessels. Postoperative hypotony increases the risk of postoperative vitreous hemorrhage as doe's preoperative neovascularization of the iris [31,32]. Previous lower extremity amputation and failure to take prescribed antihypertensives are also associated with increased risk of persistent or early vitreous cavity hemorrhage [32]. Intraoperatively, more thoroughly removing the residual peripheral vitreous gel may reduce the red blood cells seeping into the vitreous cavity and resulting in

\subsection{Cataract formation}

The existence of cataract before and after surgery in proliferative diabetic retinopathy is another important factor affecting the visual outcome. Pre-operative lenticular opacities not only affect the per-operative retinal visibility but also cause difficulty in removing the anterior vitreous from its base. All cases of dense cataract were excluded from the study. Cataract formation was occurred in two cases in group A $(6.6 \%)$, while occurred in three cases in group B (10\%). There was a slight statistical difference between the two groups which may be due to different patient ages. Two cases were complicated to silicon oil, one in each group. All cases are treated by phacoemulsification. In our study, combined phaco-vitrectomy is done in 3 cases $(10 \%)$. All are due to lens touch during surgery. persistent vitreous hemorrhage. Indeed, a small retrospective study by Cheema et al reported that 2 of 28 (7.1\%) compared with 10 of 31 (32\%) patients in the groups that respectively did and did not undergo complete removal of the residual peripheral vitreous gel had a persistent vitreous hemorrhage [24]. In our study, recurrent vitreous haemorrhage was occurred in two cases $(6.6 \%)$, one in each group $(3.3 \%)$ with no significant difference among the two groups. One case needed secondry intervention while the other was minimal bleeding which resolved spontaneously after two weeks. Studies of Mason et al [15], Guzey et al [12] and Brown et al [33] reported recurrent vitreous hemorrhage in 19\%, 9.7\% and $51 \%$ respectively. Improved results in our study are due to use of small gauge vitrectomy, meticulous vitreous removal as possible, early time of intervention shortens the period of retinal ischaemia and minimizing the production of VEGF and less neovascular proliferation and additional intra-operative endolaser photocoagulation.

Combined phaco-vitrectomy in patients having diabetic vitreous hemorrhage was reported in two different studies by Gupta et al and study of Demetriades et al as $30.34 \%, 14.05 \%$ and $28 \%$ cases respectively $[14,20,34]$. In phakic eyes in which PPV is done, cataract may develop at a rate of $20-35 \%$ post operatively [34]. In our study post-operative cataract was $16.6 \%$. Two different studies of Gupta et al [14,20], Mason et al [15] and Guzey et al [12] reported post-operative cataract formation in $20.5 \%, 22.6 \%, 15 \%$ and $9.7 \%$ respectively. This difference is because many of our patients are either young with clear lens or very old having prior cataract surgery and also due to short follow up period of our study during which cataract is not noted. 


\subsection{Postvitrectomy retinal detachment}

One of the most severe complications of PPV is postoperative retinal detachment. The retinal detachment was occurred in two cases $(6.6 \%)$, one in each group $(3.3 \%)$ with no significant difference between both groups. It can be caused by iatrogenic retinal tear, atrophic retinal tear, late contractions of vitreous incarceration and peripheral traction due to anterior hyaloid proliferation. Both cases undergone sec-

\subsection{Neovascular glaucoma}

Neovascular glaucoma was occurred in one case in group A $(3.3 \%)$, while occurred in two cases in group B (6.6\%). One case of them is one of those undergone combined phacoemulsification due to accidental lens touch. The incidence of neovascular glaucoma was more in the delayed vitrectomy group which may be

\subsection{Postoperative elevated IOP}

Postoperative elevated IOP was occurred in two cases in group A, while not occurred in group B $(0 \%)$. All cases are treated by short course of anti-glaucoma drugs including systemic and topical acetazolamide. In this study no patients in the group B had a first day IOP recorded above $21 \mathrm{mmHg}$. In group A, however, two patients $(6.6 \%)$ had a day 1 IOP above $21 \mathrm{mmHg}$ and required a short course of

\subsection{Postoperative hypotony}

Postoperative hypotony (IOP $<10$ $\mathrm{mmHg}$ ) was occurred in two cases $(6.6 \%)$, one in each group with no statistical significant diferrence among both groups. It may be caused by wound leak from sutr-

\subsection{Postvitrectomy endophthalmitis}

Post vitrectomy endophthalmitis has been reported to vary between $1.6 \%$ and $5.35 \% 25$ being nill in our study. Studies of Blankenship et al [36], Guzey et al [12] reported $0.039 \%$ and $1.39 \%$ while Mason et al [15] reported no case of post ondry intervention and treated by endolaser photocoagulation and silicon oil endotamponade. Phacoemulification and silicon oil removal was done after 3 months and the retina still attached after removal. Post vitrectomy retinal detachment as $5.5 \%$, $10.1 \%, 11 \%$ and $13.2 \%$ was reported by Guzey et al [12], Gupta et al [14,20], Mason et al [15] and Ünver et al respectively [16].

due to prolonged retinal ischaemia and release of VEGF with subsequent iris neovascularization and neovascular glaucoma. All cases are treated medically by anti-glaucoma medications and PRP and the IOP was controlled through the time of follow-up of the study.

oral acetazolamide and/or topical antiglaucoma medications. Elevation in intraocular pressure commonly occurs secondary to trabecular meshwork obstruction by erythrocytes. Eckardt made a study on 41 eyes that underwent PPV with the 23- gauge TSV system. He found that the intraocular pressure (IOP) on the first postoperative day never dropped $<12 \mathrm{mmHg}$, with no postoperative hypotony [35].

elesss clerotomies. None of the hypotonous patients developed choroidal detachment. However, at the 1-week follow-up appointment all IOPs less than $10 \mathrm{mmHg}$ had returned to within normal limits.

vitrectomy endophthalmitis. Oblique cannula insertion to facilitate scleral wound closure, and suturing any wound leaks seen following cannula removal may decrease the endophthalmitis risk[25].

\section{Conclusion}

Early vitrectomy (<30 days) with endolaser for diabetic vitreous haemorrhage decreases time spent with vision loss and the need for adjunctive PRP especially in patients of type I DM, recurrent vitreous haemorrhage and cases of premacular subhyaloid haemorrhage. Modern 
vitrectomy is safe and may be considered earlier in VH management. Visual results after PPV in diabetic vitreous hemorrhage show that most patients regain or retain useful vision. $A$ significant proportion of patients may develop late complications like recurrent vitreous hemorrhage and retinal detachment after successful PPV requiring secondary intervention.

\section{Acknowledgements}

We acknowledge all included participants, besides the clinical staff members of Ophthalmology Department, Al- Azhar University Hospital, Assiut, who helped the achievement of the study.

\section{References}

1. Klein R., Klein B., Moss S. Visual impairment in diabetes. Ophthalmology. 1984; 91: 1-9.

2. Klein R., Klein B., Moss S., et al. The Wisconsin epidemiologic study of diabetic retinopathy. IV. Diabetic macular edema. Ophthalmology. 1984; 91: 14641474.

3. The Diabetes Control and Complications Trial Research Group. The effect of intensive treatment of diabetes on the development and progression of longterm complications in insulin-dependent diabetes mellitus. N Engl J Med. 1993; 329: 977-986.

4. King, P., Peacock I., Donnelly R. The UK prospective diabetes study (UKPDS): clinical and therapeutic implications for type 2 diabetes. Br J Clin Pharmacol. 1999; 48: 643-648.

5. Flynn H., Chew E., Simons B., et al. Pars plana vitrectomy in the early treatment diabetic retinopathy study. ETDRS report number 17. The Early Treatment Diabetic Retinopathy Study Research Group. Ophthalmology. 1992; 99: 1351-1357.

6. Diabetic Retinopathy Vitrectomy Study (DRVS). Ophthalmology two-year course of visual acuity in severe proliferative diabetic retinopathy with conventional management, Report 1. 1985; 92: 492502.

7. Smiddy W., Flynn H. Vitrectomy in the management of diabetic retinopathy. Surv Ophthalmol. 1999; 43: 491-507.

8. Stefánsson E. Physiology of vitreous surgery. Graefes Arch Clin Exp Ophthalmol. 2009; 247: 147-163.

9. The Diabetic Retinopathy Vitrectomy Study (DRVS). Early vitrectomy for severe vitreous hemorrhage in diabetic retinopathy. Two-year results of a randomized trial, Report 2. 1985; 103: 1644-1652.

10. Diabetic Retinopathy Vitrectomy Study (DRVS). Early vitrectomy for severe vitreous hemorrhage in diabetic retinopathy. Four-year results of a randomized trial., Report 5. 1990; 108: 958-964.

11. Hoerauf H., Brüggemann A., Muecke M., et al. Pars planavitrectomy for diabetic macular edema. Internal limiting membrane delamination vs posterior hyaloids removal. A prospective randomized trial. Graefes Arch Clin Exp Ophthalmol. 2011; 249: 997-1008.

12. Guzey M., Müftüğlu G. Pars plana vitrectomy for high risk severe proliferative diabetic retinopathy: Anatomical and functional outcomes. Türk J Endocrinol Metab 2001; 1: 31-38.

13. Qamar R., Saleem M., Saleem M. The outcomes of pars planavitrectomy without endotamponade for tractional retinal detachment secondary to proliferative diabetic retinopathy. Int $J$ Ophthalmol. 2013; 6: 671-674.

14. Gupta A., Bansal R., Gupta V., et al. Six month visual outcome after pars planavitrectomy in proliferative diabetic retinopathy with or without a single postoperative injection of intravitreal bevacizumab. Int Ophthalmol. 2012; 32: 135-144.

15. Mason J., Colagross C., Haleman T., et al. Visual outcome and risk factors for light perception and no light perception vision after vitrectomy for diabetic retinopathy. Am J Ophthalmol. 2005; 140: 231-235.

16. Ünver Y., Yavuz G., Sinclair S. Prognostic Factors in eyes with severe proliferative diabetic retinopathy managed 
with pars plana vitrectomy. J Ret Vitr. 2009; 17: 93-100.

17. Osaadon P., Fagan X., Lifshitz T., et al. A review of anti-VEGF agents for proliferative diabetic retinopathy. Eye . 2014; 28: 510- 520.

18. La Heij E., Tecim S., Kessels A., et al. Clinical variables and their relation to visual outcome after vitrectomy in eyes with diabetic retinal traction detachment. Graefes Arch Clin Exp Ophthalmol 2004; 242: 210-217.

19. Fassbender J., Ozkok A., Hannah Canter, Schaal Sh. A comparison of early and delayed vitrectomy for management of vitreous hemorrhage due to proliferative diabetic retinopathy. Investigative Ophthalmology \& Visual Science. 2015; 56: 5117

20. Gupta B., Sivaprasad S., Wong R., et al. Visual and Anatomical outcomes follow ingvitrectomy for complications of diabetic retinopathy: The DRIVE UK Study. Eye. 2012; 26: 510-516.

21. Yorston D., Wickham L., Benson S., et al. Predictive clinical features and outcomes of vitrectomy for proliferative diabetic retinopathy. Br J Ophthalmol. 2008; 92: 365-368.

22. Khuthaila M., Hsu J., Chiang A, et al. Postoperative vitreous hemorrhage after diabetic 23-gauge pars plana vitrectomy. Am J Ophthalmol. 2013; 155: 757-763.

23. Lee B., Yu H. Vitreous hemorrhage after the 25-gauge transconjunctival sutureless vitrectomy for proliferative diabetic retinopathy. Retina. 2010; 30: 1671-1677.

24. Cheema R., Mushtaq J., Cheema M. Role of residual vitreous cortex removal in prevention of postoperative vitreous hemorrhage in diabetic vitrectomy. Int Ophthalmol. 2010; 30: 137-142.

25. Kamura Y., Sato Y., Deguchi Y., et al. Iatrogenic retinal breaks during 20-gauge vitrectomy for proliferative diabetic retinopathy. Clin Ophthalmol. 2013; 7: 29-33.

26. Landers M., Perraki A. Management of post-vitrectomy persistent vitreous hemorrhage in pseudophakic eyes. $\mathbf{A m}$ J Ophthalmol. 2003; 136: 989-993.

27. Hershberger V., Augsburger J., Hutchins R., et al. Fibrovascular ingrowth at sclerotomy sites in vitrectomized diabetic eyes with recurrent vitreous hemorrhage: Ultrasound biomicroscopy findings. Ophthalmology. 2004; 111: 1215-1221.

28. Yeh P., Yang C., Yang C., et al. Cryotherapy of the anterior retina and sclerotomy sites in diabetic vitrectomy to prevent recurrent vitreous hemorrhage: An ultrasound biomicroscopy study. Ophthalmology. 2005; 112: 2095-2102.

29. Novak M., Rice T., Michels R., et al. Vitreous hemorrhage after vitrectomy for diabetic retinopathy. Ophthalmology. 1984; 91: 1485-1489.

30. Tolentino F., Cajita V., Gancayco T., et al. Vitreous hemorrhage after closed vitrectomy for proliferative diabetic retinopathy. Ophthalmology. 1989; 96: 1495-1500.

31. Lee B., Yu H.Vitreous hemorrhage after the 25-gauge transconjunctival sutureless vitrectomy for proliferative diabetic retinopathy. Retina. 2010; 30: 1671-1677.

32. Soto-Pedre E., Hernaez-Ortega M., Vazquez J. Risk factors for postoperative hemorrhage after vitrectomy for diabetic retinopathy. Ophthalmic Epidemiol. 2005; 12: 335-341.

33. Anderson N., Fineman M., Brown G. Incidence of intraocular pressure spike and other adverse events after vitreoretinal surgery. Ophthalmology. 2006; 113 (1): 42-47.

34. Demetriades A., Gottsch J., Thomsen R., et al. Combined Phacoemulsification, intraocular lens implantation, and vitrectomy for eyes with coexisting cataract and vitreoretinal pathology. Am J Ophthalmol. 2003; 135: 291-296

35. Eckardt C. Transconjunctival sutureless 23-gauge vitrectomy. Retina. 2005; 25 (2): 208-211.

36. Blankenship G. Preoperative prognostic factors in diabetic pars plana vitrectomy. Ophthalmology. 1982; 89: 1246-1249 